\title{
Modeling hydrogen explosion in level 1 PSA
}

\author{
Julien Beaucourt, ${ }^{*}, \mathrm{a}$ and Gabriel Georgescu ${ }^{\mathrm{a}}$ \\ ${ }^{a}$ Institute of radioprotection and nuclear safety (IRSN) \\ B.P. 17 - 92262 Fontenay-aux-Roses cedex, France \\ *Email: julien.beaucourt@irsn.fr
}

Number of pages: 23

Number of tables: 0

Number of figures: 5 


\begin{abstract}
Extension of the operational lifetime beyond forty years is currently a noteworthy project in the field of nuclear safety in France, especially for 900 MWe reactors that will be the first ones to go through the fourth periodic safety review. For 1300 MWe reactors, the safety studies have already been engaged. Probabilistic safety assessments (PSA) play an important role in this process, including the assessment of the robustness of the plants against internal and external hazards. Among the hazards, internal explosion induced by hydrogen accumulation due to leakage of pipes and/or singularities, or failure of venting systems in the battery rooms is now taken into account in the PSA developped by the licensee (EDF). The PSA are then reviewed by IRSN, which is the French Technical support organization (TSO): to achieve this purpose, IRSN develops its own Level 1 PSA studies.

This paper is an updated version of the 2019 PSA contribution [1], taking into account the most recent developpments of the IRSN analyses (especially regarding the bayesian quantification of the hydrogen leak frequency derived from an original method developped by Sandia laboratories), presented at the 2019 PSAM topical conference [2].

The discussion focus on the methods related to hydrogen explosion in Level 1 PSA.The application of the methodology presented in this paper to real plant configurations is still ongoing, and the results should be presented in an other paper.
\end{abstract}

Keywords - Probabilistic safety assessment, Hydrogen explosion, Bayesian model 


\section{HYDROGEN IN PWR}

\section{I.A. Normal operation of the plant}

The use of hydrogen in Pressurized Water Reactors (PWR) is twofold. Firstly, the important heat capacity of gaseous hydrogen is used for alternator cooling. Secondly, hydrogen is also used to reduce the oxygen concentration in the Reactor Coolant System (RCS). Indeed, radiolysis effects close to the reactor core lead to production of oxygen within this system. The RCS is then voluntarily saturated with hydrogen and complex chemical recombinations allow reducing the oxygen concentration, and therefore, the oxidation of primary components.

Gaseous hydrogen is stored outside the nuclear buildings. It is directly injected into the alternator cooling system, and into the RCS through the Chemical and Volume Control System (CVCS) (see Figure 1). Primary effluents are collected within reactor and auxiliary buildings of the Nuclear Power Plant (NPP), and the gaseous effluents are stored separately in a dedicated tank before release in the atmosphere (this intermediate storage before release allows for radioactive decay). As a consequence, hydrogen circulation takes place during normal operation of the plant within nuclear auxiliary building, reactor building and turbine hall, through dedicated circuits. Hydrogen concentration within these circuits depends on the operating state, but may be as important as $100 \%$ in volume, especially for pipes directly connected to the hydrogen storage tanks. The pressure range within the pipe is usually a few bar, but some lines connected to the pressurizer are directly at the primary pressure (around 155 bar) during particular process operations.

Moreover, gaseous hydrogen is also produced by he electrical batteries, located in the electrical building. Indeed, during battery charge (following a periodical test or some particular incidental transients, e.g. external power supply of electrical boards), and floating charge, gases (oxygen and hydrogen) are produced within the batteries' cells, as a result of water hydrolysis by the charging current.

Finally, hydrogen is also produced during the treatment of seawater by electrochlorination in a pumping station ${ }^{\mathrm{a}}$. This electro-chemical process is used to produce sodium hypochlorite, which is injected into the cooling systems at the pumping station and reduces the development of

\footnotetext{
${ }^{a}$ This is true only for seaside NPP.
} 


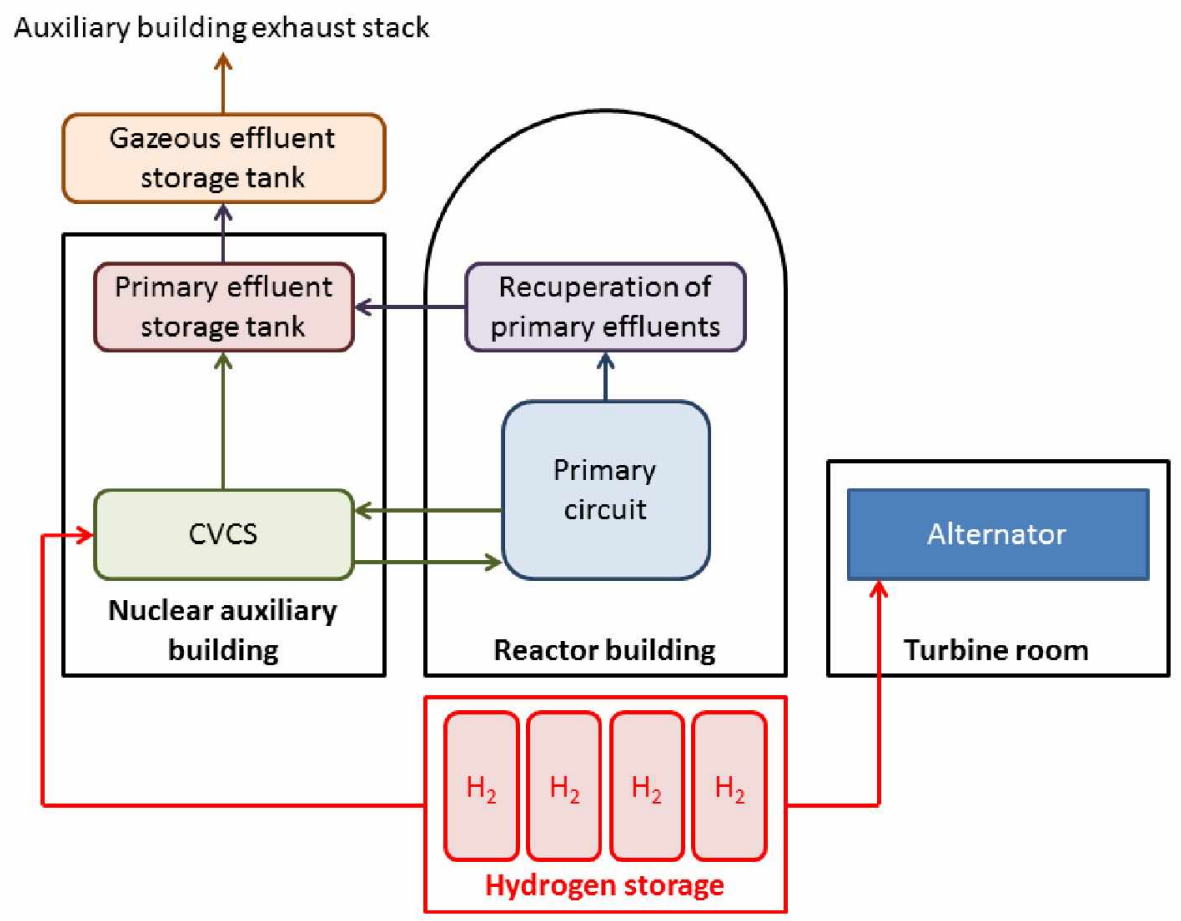

Fig. 1. Main systems involving hydrogen circulation in the nuclear island building (PWR) Simplified overview (electrochlorination device is not depicted on this figure)

biological species. This process only concerns seaside sites.

\section{I.B. Phenomenology and modeling of accidental hydrogen release}

Two situations should be considered, depending on the initiating event. Firstly, we discuss the phenomenology of hydrogen release due to a leak on a pipe or a singularity (valve, flange, etc.), and secondly, we give some general features related to the hydrogen production by electrical batteries and into the electrochlorination system.

Hydrogen is highly inflammable (the order of magnitude of the hydrogen ignition energy is $20 \mu J$ [3], which is one order of magnitude lower than "classical" hydrocarbures). Due to its high reactivity, spontaneous ignition may also occur when the temperature is important (typically when the local temperature exceeds $800 \mathrm{~K}$ [4]). The non-flammable limits, as the detonation thresholds, depend on the local relative concentration of oxygen, steam and air, and are best described by the so-called Shapiro diagram [5]. The accumulation of hydrogen in confined vented space is a complex phenomena, and some symplifying assumptions are performed by IRSN in order to assess the risk of explosion, in an efficient way, in every room of NPP. 
For the case of leaks on pipes transporting hydrogen, three situations should be considered:

- hydrogen may not inflamate at all,

- inflammation directly at the leak (immediate ignition, with probability $P_{I M}$ ), leading to a jet fire,

- accumulation within the room and detonation when the critical concentration is reached (delayed ignition, with probability $P_{D E L}$ ).

The total probability of ignition is given by the following expression:

$$
P_{i g n i t i o n}^{\text {total }}=1-\left(1-P_{I M}\right) \times\left(1-P_{D E L}\right)=P_{I M}+\left(1-P_{I M}\right) \times P_{D E L}
$$

The later situation (delayed ignition and explosion) is the most severe regarding the consequences on the NPP. The assessment of the probabilities $P_{I M}$ and $P_{D E L}$ is complex, and strongly depends on the local configuration of the leak (orientation, presence of obstacles in the neighboroud...) and the room (geometry, presence of ignition sources...). Some methods have been developped in order to assess numerically or experimentally these probabilities:

- Some models are based on a relatively simple mathematical formulation. A simplified model based on a correlation between the mass flowrate at the leak and the leak has been proposed by Cox and al. [6]. The coefficients of the correlation are essentially issued from expert judgments. The so-called RMBRA ${ }^{\mathrm{b}}$ model [7] takes into account the time exposure of the hydrogen mixture to some ignition sources, which are themselves taken into account through presence probabilities. This model is not really based on a specific caracterization of the hydrogen mixture evolution within the room, but teakes into account a heuristic exponential growth of the delayed ignition probability with time. The CCPS ${ }^{\mathrm{c}}$ model [8] takes into account experience feedback and allows for the determination of immediate and delayed ignition probabilities.

- Some time dependent sophisticated models are more and more widedly used in the offshore

\footnotetext{
${ }^{\mathrm{b}}$ Reference Manual Bevi Risk Assessment

${ }^{\mathrm{c}}$ Center for Chemical Process Safety
} 
industry: the TDIM ${ }^{\mathrm{d}}[9]$, UKOOA${ }^{\mathrm{e}}[10]$ or $\operatorname{MISOF}^{\mathrm{f}}[11]$ models may be cited. These models have been adapted in order to take into account the physical specificities of hydrogen. Regarding this constraint, some recommendations have been issued by the Hydrogen Safety (HYSAFE) network [12].

- Some analyses are also performed in order to caracterize the ignition of leaking hydrogen on an experimental basis [13].

Nevertheless, these methods are difficult to implement in an industrial framework: typically, a hundred locals have to be studied in a 900 MWe NPP, and the number of configurations to be considered is substantial. Therefore, the immediate ignition of hydrogen is not taken into account in the following, which is a conservative assumption, and only two situations are considered: absence of explosion, or explosion.

The explosion threshold is then determined using an other simplifying assumption, namely that hydrogen dispersion and accumulation occur homogeneously within the room. This may be a reasonnable assumption for small hydrogen flowrates (small leaks, hydrogen release from the batteries...) without venting, due to the high diffusity of hydrogen, but in general this is a non-conservative assumption.

Under these assumptions, it may be considered that explosion is very likely to occur (with probability equal to 1) when the hydrogen concentration in the room is higher than $4 \%$, which will be referred as the LEL (Lower Explosive Limit) in the following.

\section{IRSN METHODOLOGY FOR EXPLOSION PROBABILISTIC SAFETY AS- SESSMENT}

The use of Quantitative Risk Assessment (QRA) of hydrogen explosion hazards is relatively widespread in the offshore and hydrogen energy industry $[14,15]$. Nevertheless, these analyses are usually devoted to the determination of safety distances between hydrogen sources. The purpose of the methodology presented in this paper is different: from a nuclear safety point of view, the keypoint is to evaluate the Core Damage Frequency (CDF) induced by an explosion. Another

\footnotetext{
${ }^{\mathrm{d}}$ Time Dependent Ignition Model

eUnited Kingdom offshore operators association

${ }^{\mathrm{f}}$ Modeling of Ignition Sources on Offshore oil and gas facilities
} 
objective is to assess the efficiency of the mitigation means (venting systems, automatic isolation of hydrogen lines, etc.). More precisely, it has to be demonstrated that the frequency of non acceptable situations are very low.

IRSN has used a simplified approach for the QRA of the consequences of hydrogen explosion within nuclear island buildings. As mentioned above, during normal operation of the plant, hydrogen is circulating within the nuclear auxiliary buildings, the reactor building and the turbine hall, and is also produced permanently in the batteries rooms. Nevertheless, as a first approach, the analyses have been limited to nuclear auxiliary buildings and batteries rooms. Indeed, regarding the large volume of the reactor building and of the turbine hall, it is considered that accumulation of hydrogen above the LEL is less likely to occur.

A further probabilistic analysis of the hydrogen risk in the turbine hall should also be performed, since many support systems are transiting in this area (an explosion in the turbine hall may induce a reactor transient - turbine trip, partial or total loss of external electrical power, etc., and some mitigation means may potentially be unavailable due to the explosion - for example, some systems involved in the water supply to the emergency steam generator feedwater system may be affected by the explosion, due to the configuration of the plant). Moreover, most of the operating experience feedback on hydrogen accidental release on French NPP (see section III) took place in the turbine hall. Similarly, an analysis of the risk of explosion and its consequences should be performed for the electrochlorination process (situated in the pumping station), since an explosion in this area may induced some damages on the cooling systems of the plant.

The methodology used by IRSN for the analysis of hydrogen risk within French PWR may be decomposed in two successive steps:

- Firstly, an evaluation of the frequency of explosion is performed in every local where hydrogen pipes are present (the IRSN analysis also covers the batteries rooms, but they are not discussed in this paper): this step is described in Sec. III ;

- Secondly, the functional consequences of the explosion on the facility are evaluated : some of them are likely to initiate an accidental transient affecting the main safety function of the plant, as the protection system in such a situation (for example, the hydrogen explosion may induce a break on a pipe connected to the RCS, and simultaneously makes the safety injection system unavailable: in that case, the core meltdown is more likely to occur). The 


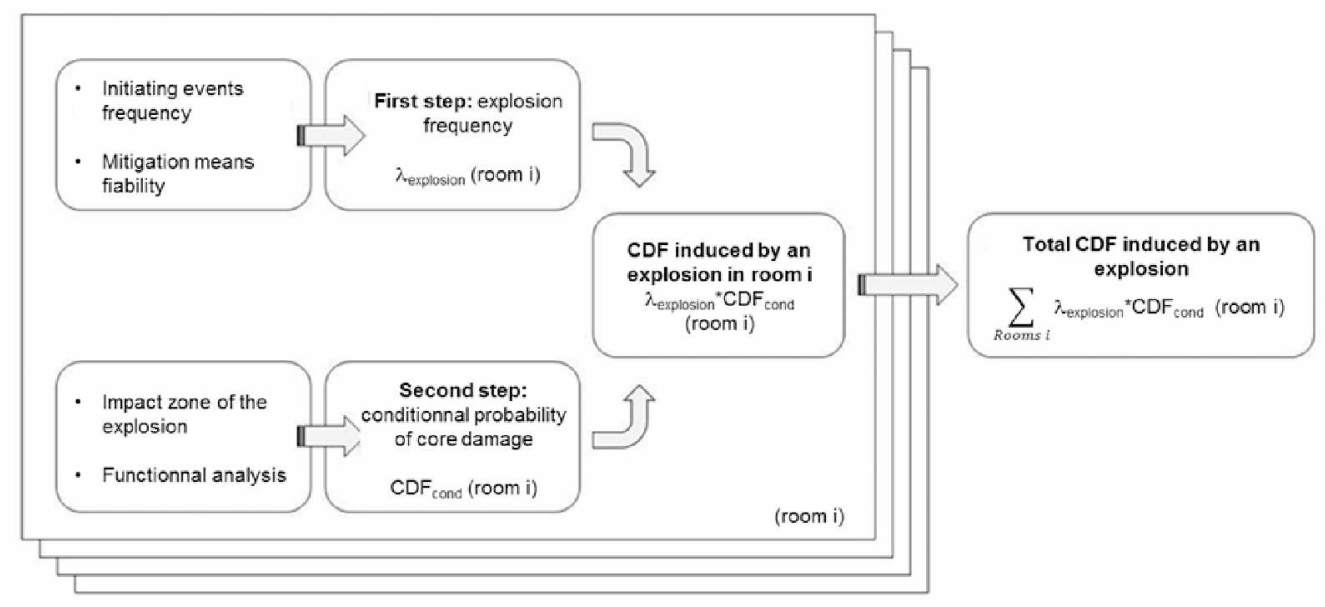

Fig. 2. Key steps of the IRSN methodology

conditional probability of core melt (assuming the occurrence of an explosion) is evaluated using the usual internal events PSA of the plant. This step is described in Sec. IV.

Finally, for every room of the auxiliary building, the risk induced by hydrogen pipe is the product of the two terms, the explosion frequency and the conditional probability of core melt. The global risk is then the sum of the risks calculated for every room.

The key steps of the methodology are summarized on Figure 2.

In the following, the discussion focus on the leaks on pipes or singularities, but the methodology may be applied to any other process leading to anormal release and accumulation of hydrogen in confined space.

\section{FIRST STEP: EVALUATION OF THE EXPLOSION FREQUENCY}

The evaluation of the frequency of explosion is itself performed in several successive steps:

- Evaluation of the hydrogen flowrate and the kinetic of hydrogen accumulation within a room: this evaluation depends on the break size (or batteries properties in the electric rooms), and is performed under the simplifying assumption that the hydrogen is homogeneously dilute within the room;

- Evaluation of the efficiency of the mitigation devices: typically, in a nuclear power plant, the two main protections against hydrogen explosion are the automatic isolation of the leak and venting systems (the main hydrogen pipes are equipped with isolation valves, and a 
closing order is automatically sent by I\&C when hydrogen concentration in the room reaches a certain threshold;

- Quantification of the explosion frequency: this evaluation is performed using simple event trees, as described hereafter, and taking into account the reliability of mitigation systems that may be efficient (depending on the break size). The key point is the evaluation of the leak frequency, as a function of its size. This evaluation is performed using a Bayesian approach, adapted from the methods used by the SANDIA laboratories [15].

\section{III.A. Hydrogen flowrate and kinetic aspects}

\section{III.A.1. Hydrogen flowrate}

\section{Leaks on pipe or singularities}

A leak on a singularity (valve, flange, etc.) or directly on a pipe leads to the accumulation of hydrogen within the room. In that case, the hydrogen flowrate at the break may be derived from the conservation of energy along a fluid line in the pipe, assuming a perfect gas relation between pressure, temperature and density, and an isentropic expansion of the hydrogen at the break. Following Cox [16], two situations should be considered according to whether the flow is subsonic or sonic. The flow should be considered as critical when the hydrogen pressure at the leak $P_{H_{2}}$ reaches a critical value $P_{c}$ given by:

$$
\frac{P_{C}}{P_{H_{2}}}=\left(\frac{2}{1+\gamma}\right)^{\frac{\gamma}{\gamma-1}}
$$

Typical values for the critical pressure $P_{c}$ are 2 bars [16]: this pressure is reached for some systems transporting gazeous hydrogen within the nuclear auxiliary building. The leak flowrate is then given by:

$$
\left\{\begin{array}{rlrl}
Q_{H_{2}}^{\text {subsonic }} & =C_{d} S \sqrt{\frac{P_{H_{2}}}{\rho_{H_{2}}} \frac{2 \gamma}{\gamma-1}\left(1-\left(\frac{P}{P_{H_{2}}}\right)^{\frac{\gamma-1}{\gamma}}\right)} \quad & \left(P_{H_{2}}<P_{c}\right) \\
Q_{H_{2}}^{\text {sonic }} & =r{ }^{\frac{P_{H_{2}}}{\rho_{H_{2}}}\left(\frac{2}{\gamma+1}\right)^{\frac{\gamma+1}{\gamma-1}}} & & \left(P_{H_{2}}>P_{c}\right)
\end{array}\right.
$$

In these equations, $P_{H_{2}}$ and $\rho_{H_{2}}$ refer to the pressure and density of hydrogen in the storage tanks. $\gamma$ corresponds to the Laplace coefficient $\gamma=1.4$ for perfect diatomic gazes). In general, the 
pressure $P$ in the room is the atmospheric pressure. $C_{d}$ is the contraction coefficient at the break (e.g. $\left.C_{d}=0.9\right)$. Some correction may be needed in order to take into account the pressure loss between the break and the hydrogen source.

\section{Batteries}

The gaseous hydrogen production in the batteries rooms is due to the charge of the batteries. Two operating modes have to be taken into account: the floating charge operating mode and the battery charge.

During the so-called floating charge operating mode, the natural self-discharge of the battery is compensated by a supply current upper than the self-discharge current. The self-discharge current of the batteries is variable in time (ageing effect) and depends on the temperature and the state of the battery. As a consequence, the floating current is not constant, but stays in the order of the milliampere/Ah.

The battery charge compensates the partial discharge or the full discharge of the battery after a situation like the loss of electrical power or a periodic test.

The accumulation of hydrogen results from the failure of the permanent venting of the battery rooms located in the electrical building. Under normal temperature and pressure conditions, and assuming that the whole electrical current applied to the battery is used for hydrogen production (which is a very conservative assumption), the hydrogen production flowrate $Q_{H_{2}}$ (in $m^{3} / h$ ) is given by Faraday's law [17]:

$$
Q_{H_{2}}^{\text {batteries }}=0.42 \times 10^{-3} \times I \times C \times N \text {. }
$$

where $\mathrm{I}$ is the applied electrical current intensity $(\mathrm{A} / \mathrm{Ah}), \mathrm{C}$ is the capacity of the elements of the battery $(\mathrm{Ah})$ and $\mathrm{N}$ is the number of elements. The order of magnitude of the charging intensity is typically $\left(10^{-4}-10^{-3}\right) \mathrm{A} / \mathrm{Ah}$. This value depends on the batteries' technology, but also on local temperature (which may be quantified using an Arrhenius law [17]), and on aging of the components.

\section{Pumping station (seaside sites)}

The treatment of seawater by electrochlorination in the pumping station consists in an alectrolysis of sea water: water molecules are decomposed into $\mathrm{H}_{2}$ and $\mathrm{OH}^{-}$ions at the cathode, and aqueous $\mathrm{Cl}^{-}$ions are combined into $\mathrm{Cl}_{2}$ gaz at the anode. The typical pure hydrogen flow rate 
produced by the process may reach several dizains of cubic meters per hour.

\section{III.B. Kinetic aspects}

For every room, it is necessary to determine the asymptotic hydrogen concentration, in order to verify if the venting system is efficient, and also the time when the critical hydrogen concentration is reached, in order to assess the efficiency of the automatic isolation of the affected pipe (when the pipe is equipped with an automatic isolation system). Assuming an homogeneous dilution of hydrogen within the room, the hydrogen concentration $C(t)$ may be evaluated using a simple linear model:

$$
\frac{d C(t)}{d t}=\frac{Q_{H_{2}}-C(t) Q_{v e n t i n g}}{V} .
$$

where $V$ stands for the volume of the local and $Q_{\text {venting }}$ for the venting flowrate in normal conditions. Eq. (5) leads to an exponential evolution of the hydrogen concentration in the local, and the asymptotic concentration results in a balance between hydrogen and venting flowrates. The LEL is reach after a time $T_{L E L}$ given by:

$$
T_{L E L}=-\frac{V}{Q_{\text {venting }}} \log \left(1-\frac{L E L \times Q_{\text {venting }}}{Q_{H_{2}}}\right)
$$

This expression is a decreasing function of the hydrogen flowrate, as expected: for a given room, increasing the hydrogen flowrate at the leak leads to a more rapid accumulation of hydrogen at the LEL.

\section{III.C. Event trees for the quantification of the explosion frequencies}

The quantification of explosion frequency within a room is performed using dedicated event trees: the initiating event is a leak on pipe, and the reliability of the different mitigation systems is modeled using classical fault trees. For every room of the auxiliary nuclear building, three different types of event trees may be constructed, depending on the leak flowrate (see Fig. 3):

- For the smallest leaks, both venting and automatic isolation may be taken into account as protection against explosion. In that case, the LEL is reached after a very long time; 


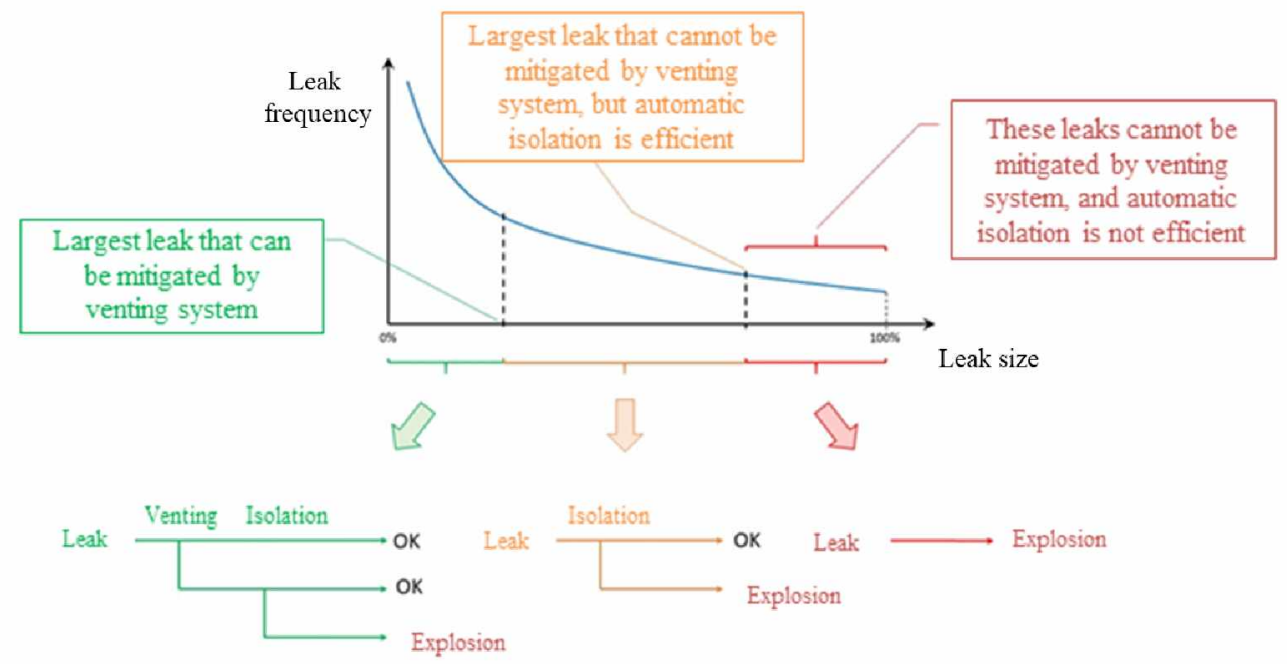

Fig. 3. Event trees for the quantification of the explosion frequency

- When the leak flowrate is more important, venting system is no more efficient and the LEL is reached in a finite time according to Eq. (6): the only protection against explosion is the automatic isolation of the line. It is important to emphasize here that the venting system of the auxiliary building has not been designed to avoid the formation of explosive atmosphere after an unexpected release of hydrogen, and as mentionned here above, the purpose of this analysis is to demonstrate that the situations that are beyond capacity of the venting system are rare enough;

- For the largest leaks, the LEL is reach so rapidly that the automatic isolation occurs too late to avoid explosion. In that case, the frequency of explosion is simply given by the frequency of the leak. Here, the situation is slightly different since this automatic isolation has been designed as a protection against explosion. Again, the purpose of this probabilistic analysis is precisely to demonstrate that the situations where this system is inefficient are rare, and have no significant consequence for the core.

Of course, depending on the local characteristics, venting system may be efficient for any break size. For some rooms, isolation may also be efficient on the whole spectrum of break sizes. And finally, in some particular situations, the automatic isolation cannot be taken into account (when the concerned hydrogen pipe is not equipped with isolation valves, or when no hydrogen detection is present in the room). These characteristics have to be listed for every room of the 
nuclear auxiliary building.

The quantification of the explosion frequency depends not only on the reliability of the mitigation provisions (which is evaluated using classical fault trees), but also on the frequency of the initiating event, namely the leak, which has to be determined for any break size.

\section{III.D. Quantification of explosion frequency}

In order to apply this methodology, it is therefore necessary to determine an analytical relationship between the break size and its occurrence frequency (see Figure 3). This relation should be possible to determine by using statistical analysis on hydrogen leaks events (on nuclear power plants or other industrial facilities). Nevertheless, most of them are considered as confidential and are not in the public domain.

The determination of the hydrogen leak frequencies is known to be a major source of uncertainties in risk analysis [14]. Some hydrogen leak events have been observed on french nuclear power plant, none of them leading to explosion within the nuclear auxiliary building. One of the difficulty when trying to use these data is to determine the break size, which is not evaluated by the licensee: most of the observed leaks are really small, and a precise evaluation of the section leak is not achievable. A qualitative description of some of these events are described in [18].

The most recent and complete study on hydrogen leak frequency determination has been performed by the Sandia laboratories [15]. The data presented in the report [15] have been used by IRSN as a starting point. A leak frequency distribution is determined for a discrete set of break size (defined as the relative section of the affected pipe: $0.01 \%, 0.1 \%, 1 \%, 10 \%$ and $100 \%$ ), using a Bayesian modeling for different categories of components (seals, valves, etc.). It is usually admitted that the determination of the prior distribution in a Bayesian modeling is a keypoint in the analysis, especially when the statistical data are sparse [19], and in order to improve the quality of the prior, the approach retained by the Sandia laboratories is sequential, in the following sense:

- In a first step, a Bayesian analysis based on a generalized linear gaussian model for the relation between the frequency and the break size is performed for data issued from different industries for hydraulic systems, denoted as "generic data" in the SANDIA report [15]. As a result, a power law relation between the leak frequency $L F$ and the break size $S$ is 


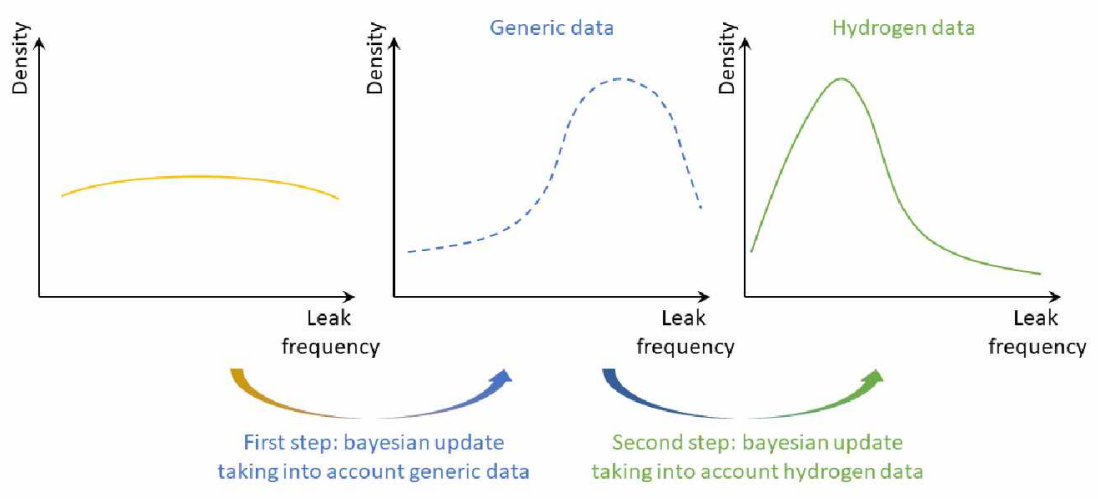

Fig. 4. Sequential Bayesian process - Simplified overview

determined for the different categories of components (seals, valves, etc.) considered in the analysis. The power law structure is the most widely used relation retained to define the relationship between break size and frequency (see for example [20] or [21]).

- This distribution is then used as a prior distribution for the evaluation of the leak frequency distribution for hydrogen components, using again a Bayesian modeling, for the leaks sizes defined here above.

This sequential Bayesian process is represented in a very schematic way on Figure 4.

As mentionned here above, a generalized linear bayesian model is used to determine the relationship between the frequency and the size of the leak [15]:

$$
\left\{\begin{array}{r}
\log \left(\mu_{j}^{g e n}\right)=\tilde{\alpha}_{0}+\tilde{\alpha}_{1} \log \left(S_{j}\right) \\
\log \left(L F_{j}^{g e n}\right) \sim \mathcal{N}\left(\log \left(\mu_{j}^{g e n}\right), \tilde{\alpha}_{2}\right)
\end{array}\right.
$$

In these equations, the subscript $j$ is an index that allows to identify the different leaks' size $(0.01 \%, 0.1 \%, 1 \%, 10 \%$ and $100 \%)$. Based on the data presented in the report [15], this model has been used by IRSN to determine the distribution of the three parameters of the model, $\tilde{\alpha}_{0}, \tilde{\alpha}_{1}$ and $\tilde{\alpha}_{2}$. The prior distribution are given by: 


$$
\left\{\begin{aligned}
& \tilde{\alpha}_{0} \sim \mathcal{N}\left(0,10^{3}\right) \\
& \tilde{\alpha}_{1} \sim \mathcal{N}\left(0,10^{3}\right) \\
& \tilde{\alpha}_{2} \sim \Gamma(1,1)
\end{aligned}\right.
$$

The results obtained by IRSN using the WinBUGS software [22] for this first step have been successfully compared to the curves presented in the report [15].

The posterior distributions for these three parameters are then used to define the prior distributions of the second step, dedicated to the analysis of hydrogen data. In the Sandia report [15], this second step is performed separetly for the different leaks sizes $S_{j}$, meaning that the power law structure is not preserved in the second step of the analysis. This approach leads to a significant reduction of the leak frequency for the break size $S_{j}$ where no hydrogen event has been recorded, and do not take into account the continuity of behavior expected for close break sizes. This continuity may be taken into account by imposing again a power law structure in the second step of the analysis, which has been performed by IRSN on the basis of the hydrogen data presented in [15]. More precisely, the same power law structure is assumed for hydrogen materials:

$$
\left\{\begin{array}{r}
\log \left(\mu_{j}^{H_{2}}\right)=\alpha_{0}+\alpha_{1} \log \left(S_{j}\right) \\
\log \left(L F_{j}^{H_{2}}\right) \sim \mathcal{N}\left(\log \left(\mu_{j}^{H_{2}}\right), \alpha_{2}\right)
\end{array}\right.
$$

and the prior distribution for the three parameters $\alpha_{0}, \alpha_{1}$ and $\alpha_{2}$ is simply the posterior distribution of $\tilde{\alpha}_{0}, \tilde{\alpha}_{1}$ and $\tilde{\alpha}_{2}$. As a result, a power law function is therefore obtained for every component taken into account in the Sandia report: this analytical relation can therefore be used as an input for the quantification of explosion frequency. An example of the results obtained by IRSN is presented on Figure 5 for valves, flanges and compressors.

These results should be used with some caution. Indeed, the hydrogen data used by Sandia are not fully presented in the report [15], where only the maximal likelihood estimator and confidence intervals are presented. The results presented on Figure 5 and summarized have been obtained by taking into account this dispersion, and not directly with the initial data. Nevertheless, the calculations presented here reveal that the methodology may be used fruitfully, and should be improved by using more specific hydrogen data.

Using the curves determined in the previous step, it is possible to determine the critical 
Seals

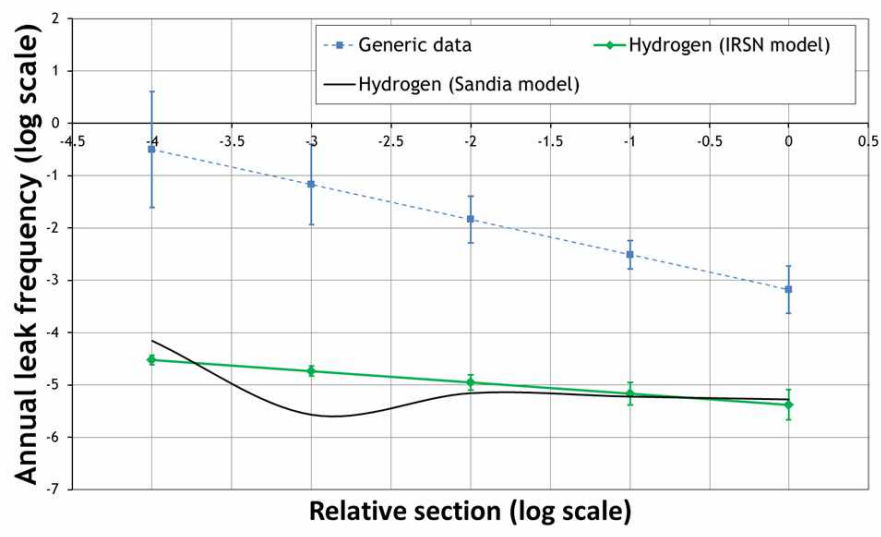

Compressors

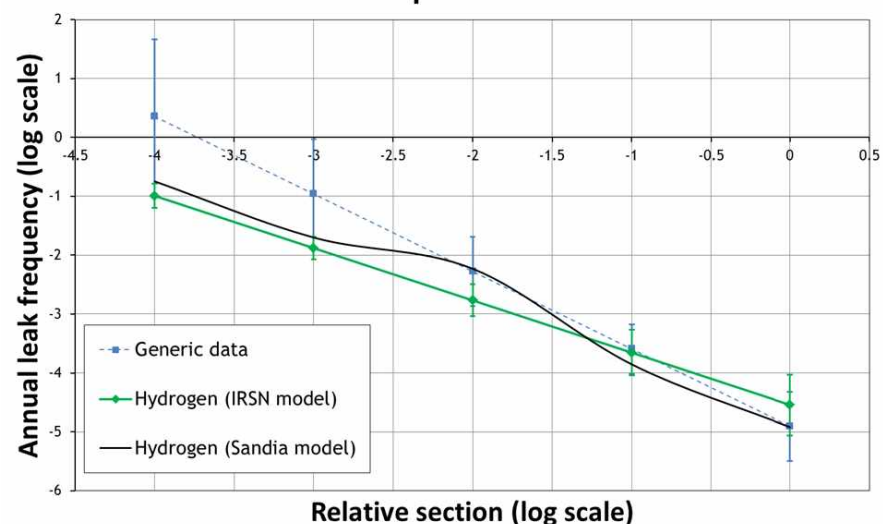

Relative section (log scale)

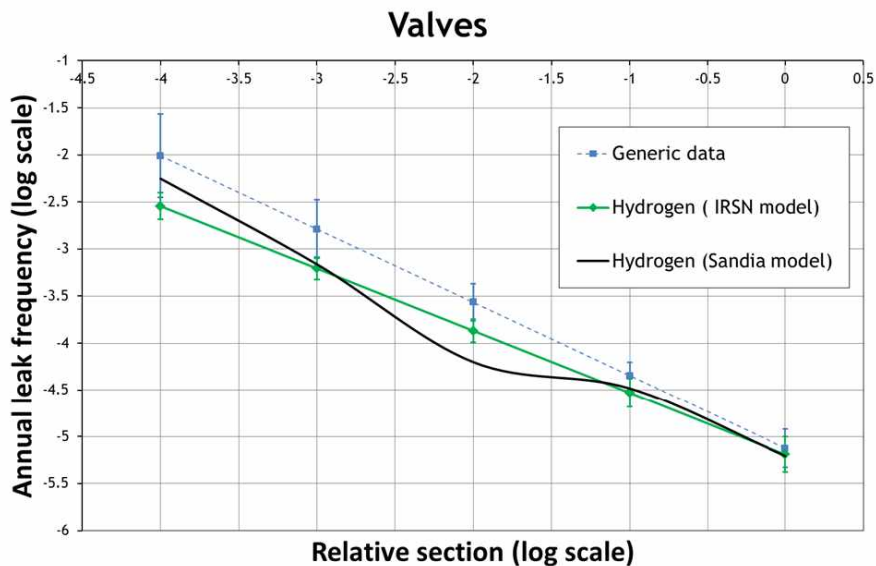

Fig. 5. Results obtained by IRSN for the second step of the sequential bayesian modeling seals; compressors; valves 
break sizes frequencies for every room of the auxiliary nuclear building. This part of the analysis is only possible if the characteristics of the rooms are known, namely the volumes, venting flowrates, number of singularities and length of hydrogen pipes. That kind of information is only possible to obtain through plant walkdowns, which have been organized by IRSN with the licensee support.

At the end of this first step, a frequency of explosion for every room in the auxiliary nuclear building is determined, using the specific fault trees presented on Figure 3. The establishment of a first hierarchy of the rooms, regarding the risk of explosion, is therefore possible. As pointed here above, this classification is not sufficient by itself for nuclear safety analysis, and the consequences of the explosion on the plant have to be somehow evaluated.

\section{SECOND STEP: CONSEQUENCES OF THE EXPLOSION ON THE FACIL- ITY}

\section{IV.A. Determination of the impact zones, functional analysis}

For every possible source of accidental hydrogen release, it is necessary to evaluate the impact zone of the explosion. This is a two phases' step: firstly, the extent of the zone affected by the explosion has to be determined, and secondly, it is necessary to evaluate the consequences of the explosion on the safety equipment located in the environment.

The evaluation of the impact zone is clearly a difficult task. As a first approach, IRSN considered that only the room where the hydrogen leak occurs is affected. This is obviously a non conservative assumption, due to the fact that the rooms in the nuclear auxiliary building are interconnected: the different rooms are generally separated by fire doors, which are not necessarily designed to support overpressure resulting from the explosion of a confined hydrogen mixture. Some sensitivity analyses have been performed, in order to evaluate the dispersion of hydrogen mixture in the surrounding rooms, and to determine, for some limited cases, if the LEL could be reached beyond the room where the leak occurred.

Basically, the functional analysis consists in an evaluation of the damages induced by the explosion in the facility, and more precisely, on the System, Structure and Component (SSC) that are taken into account in the level 1 PSA for internal events. In the impact zone defined here above, all the initiating events (as defined in the level 1 PSA for internal events) that may be induced by explosion, as the SSC that are taken into account to cope with these particular initiating events, 
are listed. In principle, a mechanical analysis of the explosion consequences should be performed for all of these SSC. But this analysis is complex, since a precise 3D modeling of the geometrical configuration of all the rooms of the nuclear auxiliary building is not reasonably achievable (as mentioned before, several hundreds of possible sources of hydrogen release should be analyzed). Moreover, the physical modeling of the propagation of the shock wave, and the estimation of the damages induced on the SSC located in the area, is very challenging. Therefore, it is considered that all the SSC located in the impact zone are lost due to the explosion. It is believed however that this assumption is not necessarily over conservative, due to the violence of the involved phenomena (shock waves, high temperature front and fire propagation, etc.).

\section{IV.B. Quantification of the induced core damage frequencies}

The PSA for internal events is finally used to evaluate the induced core damage frequencies. The induced core damage frequency is evaluated as the sum of the contribution of all possible sources in all considered rooms:

$$
C D F_{\text {explosion }}=\sum_{\text {roomsi }} \lambda_{\text {roomi }}^{\text {explosion }} C D F_{\text {roomi }}
$$

where $\lambda_{\text {roomi }}^{\text {explosion }}$ corresponds to the explosion frequency in the room, and $C D F_{\text {roomi }}$ is the induced core damage frequency (CDF) by an explosion in the considered room $i$.

Finally, a second hierarchy of the rooms is determined, which may differ from the one obtained at the end of step 1 Sec. III.

\section{CONCLUSION AND PERSPECTIVES}

IRSN modeling of the explosion consequences on the SSC located in the auxiliary nuclear building and in the electrical buildings was used as a support for the review of the corresponding EDF studies, performed in the framework of the forth Periodic Safety Review (PSR) of the French 900 MWe NPP. In this paper, a general discussion on the determination of the input data has been presented. Using an analytical relation between the break size and the occurrence frequency allows to use a more efficient approach that the one that has been presented by IRSN in the 2019 PSA conference [1]. 
Despite some simplifying assumptions, this model captures the main features of the risks induced by the explosion of a hydrogen mixture. An important outcome of this analysis is that the most important contributors to the global risk induced by explosion are not necessarily the rooms where the evaluated frequency of explosion is the most important. The analysis also revealed that functional analysis is a cornerstone of the evaluation. Moreover, the analysis outcomes allow establishing a hierarchy between the different rooms, in terms of core damage frequency. As mentioned here above, one of the outcome of this analysis is to demonstrate the robustness of the protection systems, especially the automatic isolation of the hydrogen pipes when a hydrogen release is detected. The situations where this system is not sufficient and lead to an explosion and to core damage should be rare. For the most important rooms, existing deterministic analysis may be complemented, for example to evaluate the robustness of the plant for hydrogen risk when specific deterministic rules are taken into account for the mitigation systems: venting, hydrogen detection, etc. (as for example, additional failure criteria). Also, the general operating rules may take into account some reinforced requirements for the most important rooms (technical specifications, periodic inspection of the venting system, etc.).

Nevertheless, the IRSN PSA modeling of internal explosion consequences is based on some simplifying, and sometimes possible non conservative assumptions. In particular, the hypothesis of homogeneous dilution of hydrogen in case of pipe leak or the loss of venting in the electrical batteries room may be reviewed for some particular geometric configuration of the rooms. On the other side, the lack of conservatism of some assumptions is difficult to assess without any quantitative evaluation. Typically, the assumption that the hydrogen release do not spread to other rooms than the one where the leak is located should be, and will be, questioned. Indeed, the different rooms in the auxiliary nuclear building are usually interconnected through the venting system. This effect is not taken into account in the present study, and it is difficult to determine if this simplified assumption is conservative or not. Dilution of hydrogen is connected rooms by the venting system may avoid the formation of explosive mixture, but on the other side, if the hydrogen leak is important, the LEL may be reached in several rooms.

In the next future, these two assumptions (homogeneous dilution and hydrogen spreading through the connected rooms) will be questioned by IRSN, in order to evaluate more precisely the dispersion of hydrogen and the extension zone of the damages. Moreover, the statistical 
evaluations of the leak frequencies will be improved, by aggregating data issued from the french operating experience feedback, and by optimizing the Bayesian prior determination. Finally, the application of this methodology to real plant configuration is still an ongoing process, and the results will be presented and discussed in a future paper. 


\section{ACKNOWLEDGMENTS}

The authors wish to thank Angélique Pasco and Marc Henrio (IRSN) for precious support during on-site walkdown. 


\section{REFERENCES}

[1] J. Beaucourt and G. Georgescu, "Modeling hydrogen explosion in level 1 PSA," 16th ANS International Topical Meeting on Probabilistic Safety Analysis and Analysis (PSA 2019), Charleston Marriott, South Carolina USA, American Nuclear Society (2019).

[2] J. Beaucourt, A. Pasco, and G. Georgescu, "Toward a risk informed hierzrchy of hydrogen rooms in nuclear power plants," Practical Use of Probabilistic Safety Assessment in Operations (PSAM topical 2019), The Haymarket Hotel, Stockholm Sweden, International association for probabilistic safety assessment and management (IAPSAM), Lloyd's register consulting (2019).

[3] A. J. Metzler, "Minimum ignition energies of six pure hydrocarbon fuels," RM E52 F27, National advisory committee for aeronautics (NACA) (1952).

[4] G. R. Colonna, "Fire protection guide to hazardous materials," NFPA 704, National fire protection association (2010).

[5] D. JACQUEMaIn, Nuclear power reactor core melt accidents - Current state of knowledge, EDP Sciences (France) (2015).

[6] A. W. Cox, F. P. LEEs, and M. L. ANG, "Classification of hazardous locations," Institution of chemical engineers (1990).

[7] "Reference Manual Bevi Risk Assessment," Version 3.2, National institute of public health and the environment (RIVM) (2009).

[8] "Guidelines for determining the probability of ignition of a released flammable mass,", Center for Chemical Process Safety (2014).

[9] E. M. Berg, A. Huser, and E. Skramstad, "Ignition modelling - Time dependent ignition probability model," 96-3629 rev. 04, DNV research \& innovation - Joint industry project (JIP) (1996).

[10] "Ignition probability review, model development and look-up correlations - IP research report," ISBN 978085293454 8, Energy institute (2018). 
[11] I. Fossan, A. O. Sæ Bo, H. O. R., and M. K., "Modelling of ignition sources on offshore oil and gas facilities - MISOF(2)," 107566/R2, Loyd's register consulting (2018).

[12] "An ignition probability model methodology for hydrogen risk analysis," D71, HYSAFE (2007).

[13] M. R. Swain, P. A. Filoso, and M. N. Swain, "An experimental investigation into the ignition of leaking hydrogen," International Journal of Hydrogen Energy, 32, 2, 287 (2018).

[14] "Main report - Survey of hydrogen risk assessment methods," 2005-1621 rev.2, DNV research \& innovation (2008).

[15] J. Lachance, W. Houf, B. Middleton, and F. L., "Analyses to support development of risk-informed separation distances for hydrogen codes and standards," SAND2009-0874, SANDIA national laboratories (2009).

[16] A. W. Cox, "Studies in support of a quantitative approach to hazardous area classification," PhD Thesis, Loughborough University (1989).

[17] "Safety requirements for secondary batteries and battery installations," EN 50272, European standard (2001).

[18] "Experience feedback on hydrogen hazards in 900 MWe PWRs," 7977, IAEA - International reporting system for operating experience (IRS) (2008).

[19] C. ATwood and AL., "Handbook of parameter estimation for probabilistic risk assessment," SAND2003-3348P (NUREG/CR-6823), SANDIA national laboratories (2003).

[20] J. Spouge, "New generic leak frequencies for process equipment," Process safety progress, $24,249(2005)$.

[21] R. Pitblado and AL., "Frequency data and modification factors used in QRA studies," Journal of loss prevention in the process industries, 24, 3, 249 (2011).

[22] D. Lunn, C. Jackson, N. Best, A. Thomas, and D. Spiegelhalter, The BUGS BookA Practical Introduction to Bayesian Analysis, CRC Press / Chapman and Hall (2012). 


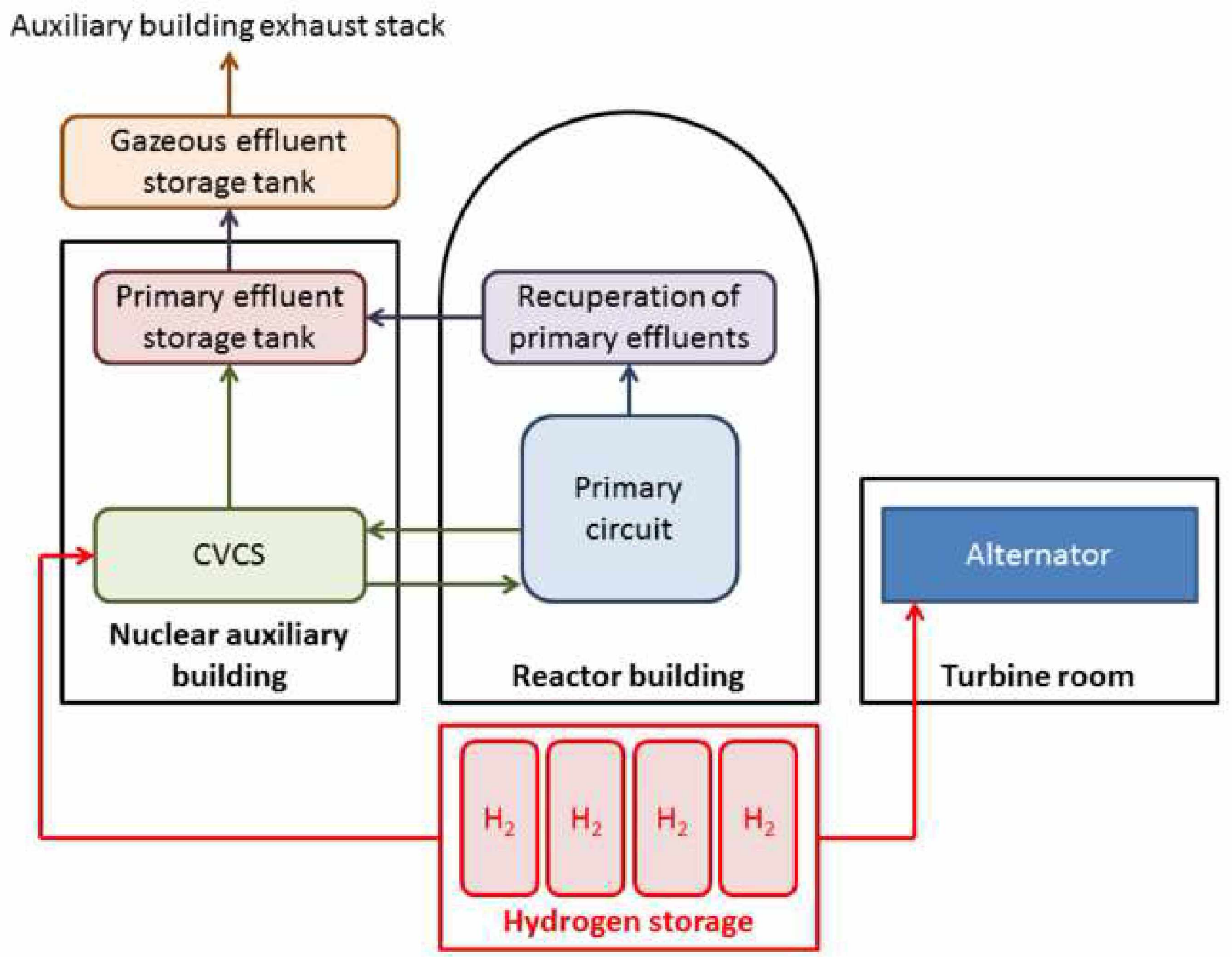




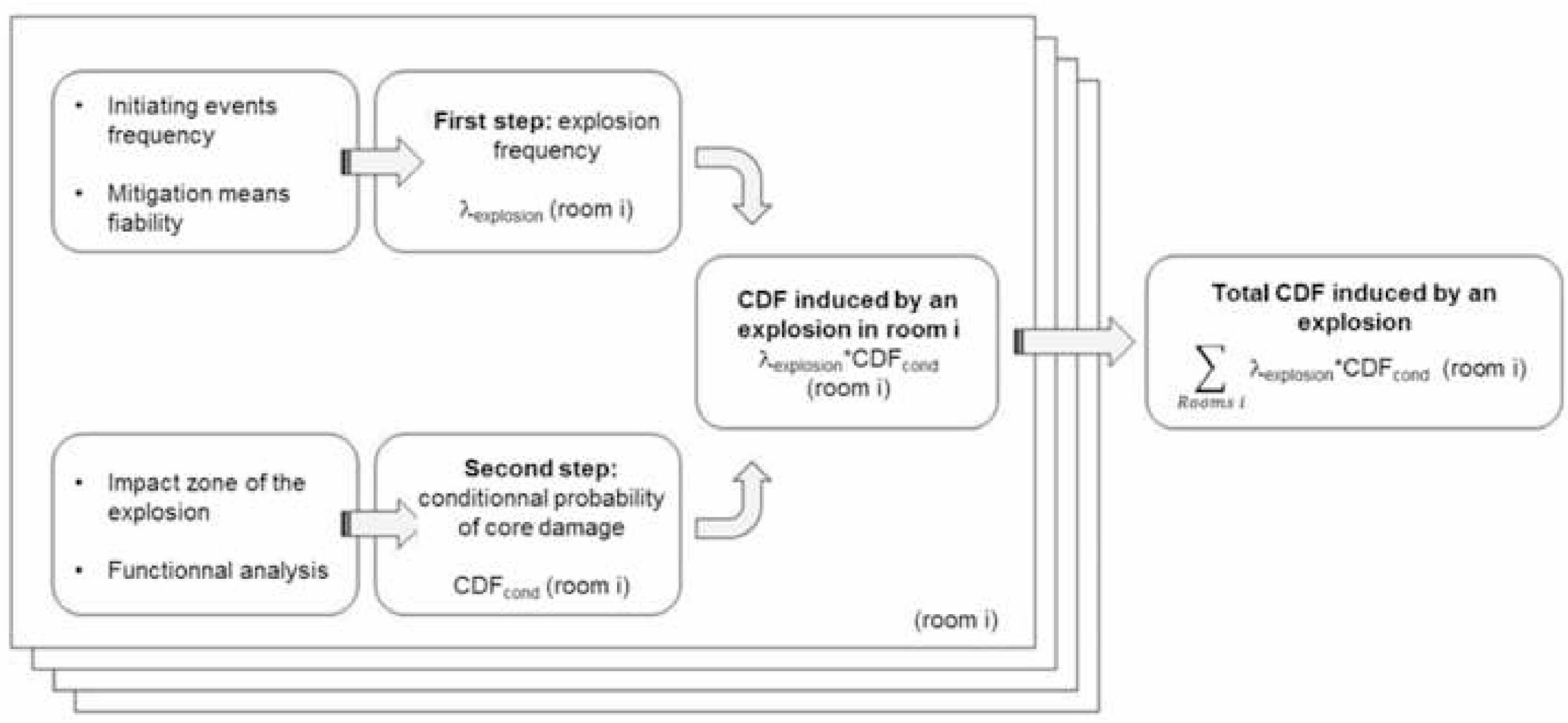




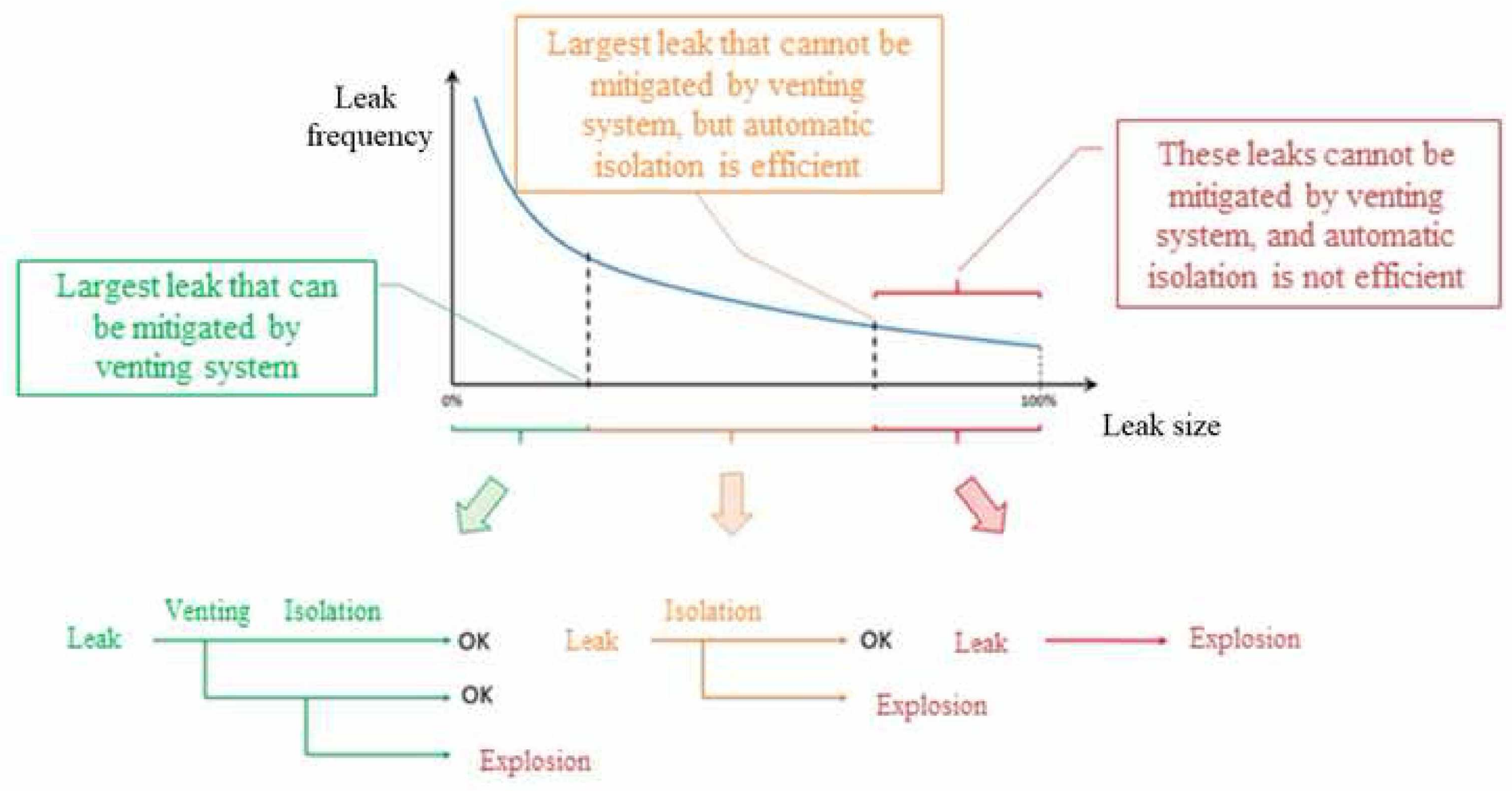



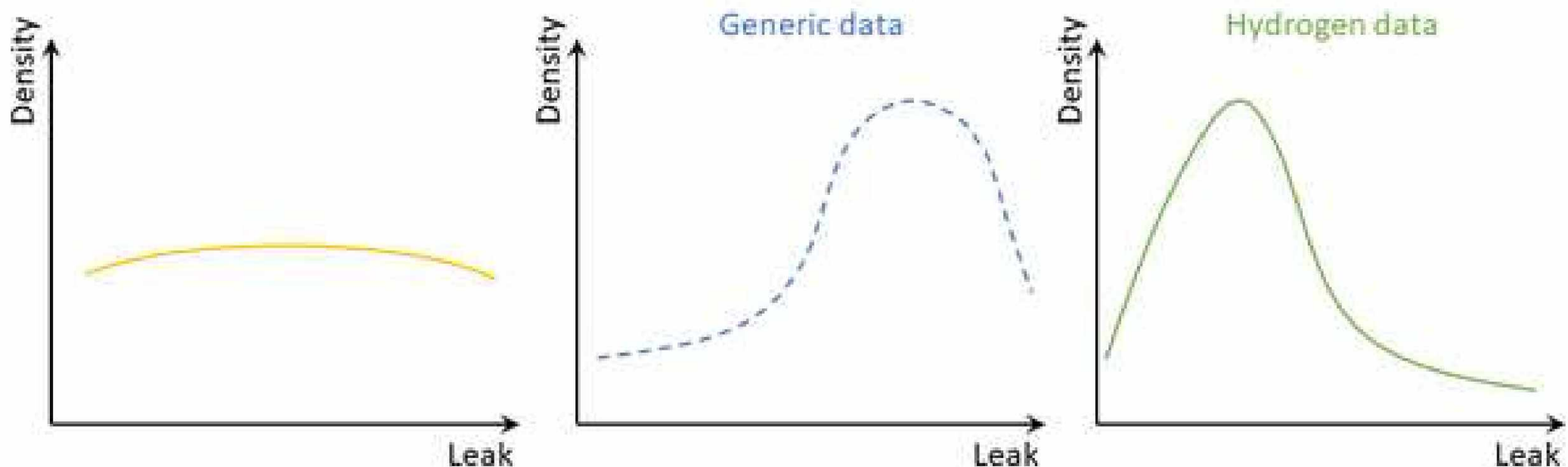

frequency

frequency

frequency

First step: bayeșian update

taking into account generic data
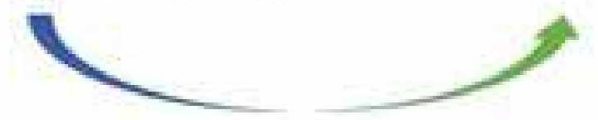

Second step: bayesian update

taking into account hydrogen data 


\section{Seals}

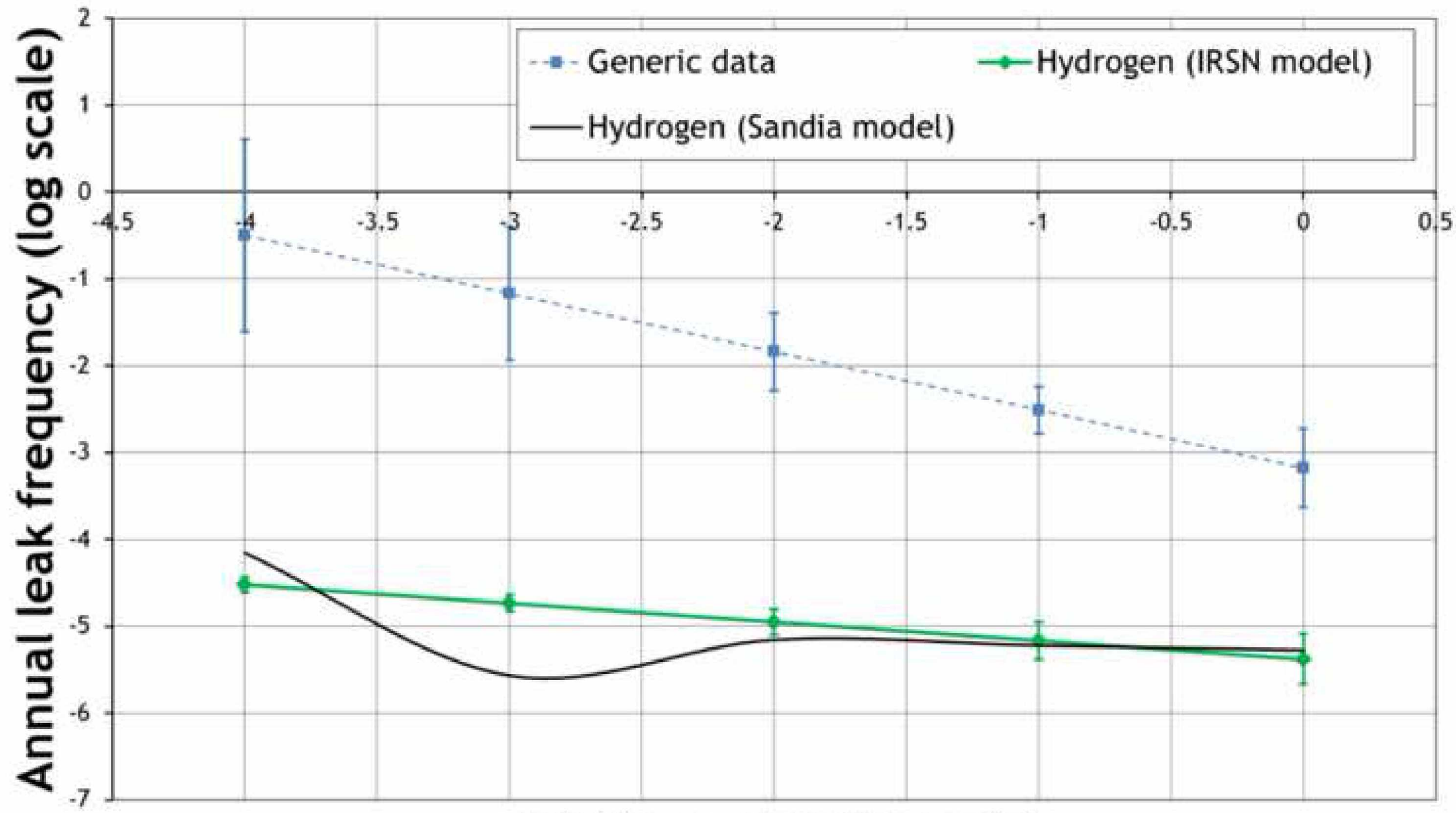

\section{Relative section (log scale)}




\section{Compressors}

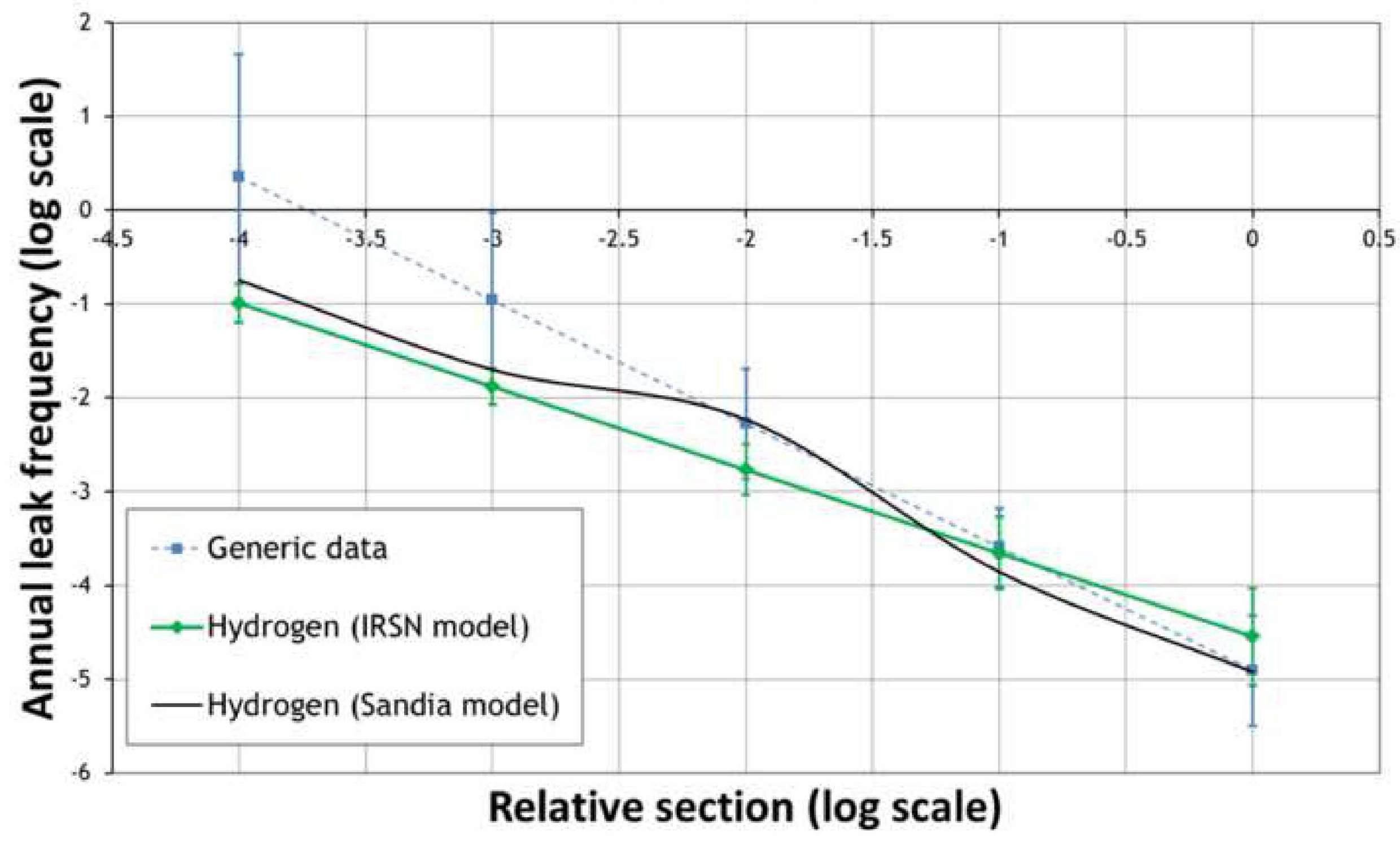


Annual leak frequency (log scale)

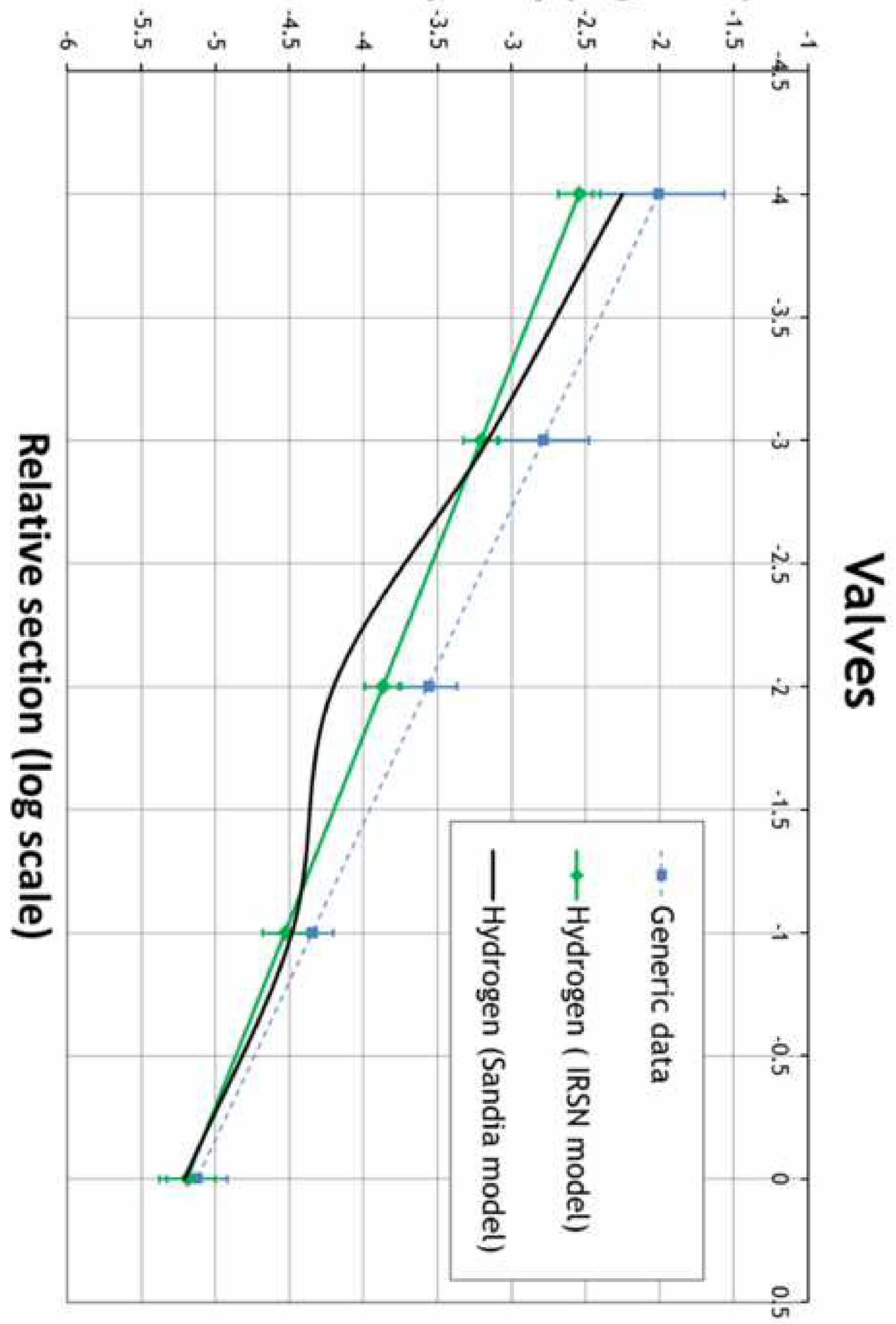

